\title{
Lung fluid transport in aquaporin-5 knockout mice
}

\author{
Tonghui Ma, Norimasa Fukuda, Yuanlin Song, Michael A. Matthay, and A.S. Verkman
}

Departments of Medicine and Physiology, Cardiovascular Research Institute, University of California, San Francisco, California 94143, USA

Address correspondence to: Alan S. Verkman, Cardiovascular Research Institute, 1246 Health Sciences East Tower, Box 0521, University of California San Francisco, San Francisco, California 94143-0521, USA.

Phone: (415) 476-8530; Fax: (415) 665-3847; E-mail: verkman@itsa.ucsf.edu; Website: http://www.ucsf.edu/verklab.

Tonghui Ma, Norimasa Fukuda, and Yuanlin Song contributed equally to this work.

Received for publication August 25, 1999, and accepted in revised form November 9, 1999.

The mammalian lung expresses water channel aquaporin-1 (AQP1) in microvascular endothelia, AQP4 in airway epithelia, and AQP5 at the apical plasma membrane in type I cells of alveolar epithelia. We previously studied the role of AQP1 and AQP4 in lung fluid transport using knockout mice. Here, we examined the role of AQP5 using AQP5 knockout mice, which were recently shown to manifest defective saliva secretion. $A Q P 5$ deletion did not affect lung morphology at the light microscopic level, nor did it affect the distribution or expression of aquaporins 1, 3, or 4. Airspace-capillary osmotic water permeability $\left(\mathrm{P}_{\mathrm{f}}\right)$ was measured in isolated perfused lungs by pleural surface fluorescence and gravimetric methods. $\mathrm{P}_{\mathrm{f}}$ was reduced 10 -fold by $A Q P 5$ deletion and was further reduced by 2 - to 3 -fold in $A Q P 1 / A Q P 5$ double-knockout mice. Hydrostatic lung edema in response to acute increases in pulmonary artery pressure was not affected by $A Q P 5$ deletion. Active alveolar fluid absorption was measured in an in situ lung model from the increase in concentration of a volume marker in an isosmolar alveolar instillate. Interestingly, fluid absorption did not differ in litter-matched AQP5 knockout mice, nor was there an effect of AQP5 deletion when fluid absorption was maximally stimulated by pretreatment of mice with keratinocyte growth factor. These results indicate that AQP5 is responsible for the majority of water transport across the apical membrane of type I alveolar epithelial cells. The unimpaired alveolar fluid clearance in AQP5-null mice indicates that high alveolar water permeability is not required for active, near-isosmolar fluid transport.

J. Clin. Invest. 105:93-100 (2000).

\section{Introduction}

The lung has exceptionally high epithelial and endothelial water permeability (1). Very rapid osmotically driven water transport between the airspace and capillary compartments has been found in several mammals, including mouse, rat, rabbit, and sheep (2-4). The vast majority of the airspace surface consists of alveolar type I epithelial cells. In isolation, type I cells have the highest water permeability of any mammalian cell type studied to date (5). Movement of water from the airspace to the alveolar capillaries also requires transit across the microvascular endothelium, which also is highly water permeable (6). The epithelium lining the airways, which makes up a relatively small fraction of the total airway surface, has substantially lower osmotic water permeability $\left(\mathrm{P}_{\mathrm{f}}\right)$ than does the alveolar epithelium or the microvascular endothelium (7). It is believed that high lung water permeability facilitates fluid absorption in the perinatal lung and airspace hydration in the adult lung, as well as the formation and resolution of pulmonary edema $(8,9)$.

Several aquaporin-type water channels are expressed in lung and are thought to be responsible for the high lung water permeability. The aquaporins are a conserved family of small water-transporting proteins, 10 of which have been identified in mammals with numerous homologues in lower animals and plants. Three aquaporins have been located in lung: AQP1 in microvascular endothelia and some pneumocytes (2, 10-12), AQP4 in the basolateral membrane of airway epithelium $(13,14)$, and AQP5 in the apical membrane of type I alveolar epithelial cells $(14,15)$. A fourth aquaporin, AQP3, is expressed in trachea and large airways but not in peripheral lung $(10,13)$. Expression of AQP4 and to a lesser extent AQP1 increases sharply around the time of birth (16-18), and lung aquaporin expression appears to be regulated by various factors such as corticosteroids, keratinocyte growth factor (KGF), and lung injury $(19,20)$.

We recently generated knockout mice lacking the 3 lung water channels, each of which also is expressed outside the lung. AQP1 knockout mice are unable to generate concentrated urine (21) because of defective isosmolar fluid absorption in proximal tubule (22) and defective medullary countercurrent exchange (23). In lung, AQP1 deletion produced a decrease of more than 10-fold in microvascular and airspace-capillary $\mathrm{P}_{\mathrm{f}}$, as well as a small decrease in hydrostatically driven lung edema (24). AQP4 knockout mice manifest a minor defect in urinary concentrating ability (25) resulting from a 4-fold reduction of water permeability in inner 
medullary collecting duct (26). Also, these mice are protected against cerebral edema in response to acute water intoxication and ischemic stoke (27). In lung, AQP4 deletion did not affect water permeability or alveolar fluid clearance (24). AQP5 is a water-selective channel expressed in type I alveolar cells and in the apical membranes of serous acinar cells in salivary glands, lacrimal glands, and surface corneal epithelial cells (10, $14,15,28)$. We recently generated AQP5-deficient mice. These mice manifest defective agonist-stimulated saliva secretion, with the production of small quantities of viscous hypertonic saliva (29). The defect in saliva secretion in AQP5 knockout mice and the defect in proximal tubule fluid absorption in AQP1 knockout mice support the paradigm that high plasma membrane water permeability is required for active, near-isosmolar fluid absorption and secretion across epithelia (30).

The above considerations predict that AQP5 may be critical to both passive and active lung fluid transport. We report here a comparison of osmotic water transport, hydrostatic filtration, and active alveolar fluid absorption in wild-type and AQP5 knockout mice. AQP5 deletion produced a dramatic decrease in airspace-capillary $\mathrm{P}_{\mathrm{f}}$, but did not affect active alveolar fluid absorption, even when active fluid absorption was maximally stimulated by $\beta$-agonists and KGF pretreatment. These results have important implications regarding the role of high alveolar epithelial water permeability and AQP5 in lung physiology.

\section{Methods}

Transgenic mice. Transgenic knockout mice deficient in AQP5 were generated by targeted gene disruption as described recently (29). Measurements were done in litter-matched mice (8-10 weeks of age; $20-25$ grams in body weight) produced by intercrossing CD1 heterozygotes. AQP1/AQP5 double-knockout mice were generated by serial breeding of AQP1 and AQP5 knockout mice to yield double-heterozygous mice and then double-knockout mice. Mice were housed in air-filtered, temperature-controlled units with free access to food and water. Genotype analysis of tail DNA was done by PCR at age 5 days. To date, 443 mice have been born from breeding AQP5 heterozygous mice with a genotype distribution of 187:171:85 wild-type/heterozygous/knockout. The investigators were blinded to genotype information for all transport measurements. Protocols were approved by the University of California San Francisco Committee on Animal Research.

Isolated lung perfusion. Mice were euthanized with intraperitoneal pentobarbital $(150 \mathrm{mg} / \mathrm{kg})$. The trachea was cannulated with polyethylene PE-90 tubing and the pulmonary artery with PE-20 tubing. The left atrium was either transected or cannulated with PE-20 tubing. The heart and lungs were moved en bloc to a perfusion chamber for observation by epifluorescence microscopy, or to a gravimetric apparatus (see below). The pulmonary artery was gravity perfused at constant pressure at room temperature. For measurement of air- space-capillary water permeability, the airspace was filled with $0.5 \mathrm{~mL}$ HEPES-buffered Ringer's (HBR) solution $(137 \mathrm{mM} \mathrm{NaCl}, 2.68 \mathrm{mM} \mathrm{KCl}, 1.25 \mathrm{mM}$ $\mathrm{MgSO}_{4}, 1.82 \mathrm{mM} \mathrm{CaCl}, 5.5 \mathrm{mM}$ glucose, $12 \mathrm{mM}$ HEPES, and 5\% BSA; pH 7.4; 300 mOsm) containing (for surface fluorescence measurements) FITC-dextran $(70 \mathrm{kDa} ; 0.5 \mathrm{mg} / \mathrm{mL})$. The fluorescence intensity from a spot $3-5 \mathrm{~mm}$ in diameter on the lung pleural surface was monitored with an inverted epifluorescence microscope using a fluorescein filter set.

Pleural surface measurement of osmotic water permeability. For measurement of airspace-capillary $\mathrm{P}_{\mathrm{f}}$, an airspaceto-perfusate osmotic gradient was generated by switching the perfusate between HBR and either HBR containing $200 \mathrm{mM}$ sucrose (500 mOsm) or HBR diluted with water to $200 \mathrm{mOsm}$. The time course of pleural surface fluorescence was monitored continuously. $\mathrm{P}_{\mathrm{f}}$ was computed using the equation:

\section{Equation 1}

$$
P_{\mathrm{f}}=\left[\mathrm{d}\left(\mathrm{F} / \mathrm{F}_{\mathrm{o}}\right) / \mathrm{dt}\right]_{\mathrm{t}=0} /\left[\left(\mathrm{S} / \mathrm{N}_{\mathrm{o}}\right) v_{\mathrm{w}} \Delta \mathrm{C}\right]
$$

where $P_{f}$ is the osmotic water permeability coefficient (in $\mathrm{cm} / \mathrm{s}),\left[\mathrm{d}\left(\mathrm{F} / \mathrm{F}_{\mathrm{o}}\right) / \mathrm{dt}\right]_{\mathrm{t}=0}$ is the initial slope of the relative fluorescence vs. time data, $\mathrm{S} / \mathrm{V}_{\mathrm{o}}$ is surface-to-volume ratio in $\mathrm{cm}^{-1}, \mathrm{v}_{\mathrm{w}}\left(18 \mathrm{~cm}^{3} / \mathrm{mol}\right)$ is the partial molar volume of water, and $\Delta \mathrm{C}$ is the difference in osmolality between perfusate and airspace fluids (3).

Gravimetric measurement of osmotic water permeability and filtration. A gravimetric method was used to continuously monitor water content in the isolated perfused lung. Lungs were suspended from a weight transducer (sensitivity $<1 \mathrm{mg}$ ) and perfused as described above. The cumulative weight of fluid exiting the lung was recorded continuously using a weight transducer to determine perfusion rate. The pulmonary artery was gravity perfused with HBR. The trachea was transected and left open to the air. For measurement of $\mathrm{P}_{\mathrm{f}}$, the time course of lung weight was determined in response to changes in pulmonary artery perfusate osmolality from $300 \mathrm{mOsm}$ to $500 \mathrm{mOsm}$, or from $300 \mathrm{mOsm}$ to $200 \mathrm{mOsm}$. For measurement of hydrostatically driven edema (filtration), lung weight was measured in response to an increase in pulmonary artery perfusate pressure from 8 $\mathrm{cm} \mathrm{H}_{2} \mathrm{O}$ to $18 \mathrm{~cm} \mathrm{H}_{2} \mathrm{O}$, and then to $38 \mathrm{~cm} \mathrm{H}_{2} \mathrm{O}$ at a constant left atrial outflow pressure of $5 \mathrm{~cm} \mathrm{H}_{2} \mathrm{O}$. Lung weight and perfusate flow were recorded by gravimetric transducers and an analog-to-digital computer interface (Biopak Inc., Santa Barbara, California, USA).

Measurement of alveolar fluid clearance. Mice were euthanized as described above and were maintained at $37-38^{\circ} \mathrm{C}$ using a heating pad, an infrared lamp, and an intra-abdominal monitoring thermistor. The trachea was cannulated with a 20-gauge trimmed Angiocath catheter (Becton Dickinson, Sandy, Utah, USA) through a tracheostomy. The lungs were inflated with $100 \% \mathrm{O}_{2}$ at $7 \mathrm{~cm} \mathrm{H}_{2} \mathrm{O}$ continuous positive airway pressure throughout the experiment. To measure alveolar fluid clearance, $10-13 \mathrm{~mL} / \mathrm{kg}$ of instillate was delivered to both lungs over 30 seconds through the tracheal can- 
nula. An alveolar fluid sample $(50-100 \mu \mathrm{L})$ was aspirated with a $1-\mathrm{mL}$ syringe connected directly to the catheter. Lungs were removed by a median sternotomy, and a blood sample was obtained from the heart. The aspirate was centrifuged at $3,000 \mathrm{~g}$ for 10 minutes, and the supernatant was assayed for protein concentration and radioactivity.

The instillate consisted of Ringer's lactate containing BSA $(5 \mathrm{~g}$ per $100 \mathrm{~mL})$ and ${ }^{131} \mathrm{I}$-albumin $(0.1 \mu \mathrm{Ci}$; MerckFrosst Canada, Montreal, Quebec, Canada) adjusted to $340 \mathrm{mOsm}$ with $\mathrm{NaCl}$ to match the measured mouse serum osmolality of $336 \pm 12$ mOsm (mean \pm SD, $n=$ 15). In some experiments, isoproterenol, terbutaline, amiloride, or ouabain was added to the instillate. The percentage fluid absorption at 15 minutes was computed from the ratio of instilled and sample radioactivities as described previously $(24,31)$. Alveolar fluid clearance (AFC) was computed from the increase in alveolar fluid albumin using the equation:

\section{Equation 2}

$A F C=\left(V_{i} F_{w i}-V_{f} F_{w i}\right) / 100 V_{i} F_{w i}$

where $F_{w}$ is the water fraction of the initial (i) and final (f) alveolar fluid determined gravimetrically, $V_{\mathrm{i}}$ is initial alveolar fluid volume, and $V_{\mathrm{f}}$ is final alveolar fluid volume, determined from:

\section{Equation 3}

$\mathrm{V}_{\mathrm{f}}=\mathrm{V}_{\mathrm{i}} \mathrm{TP}_{\mathrm{i}} \mathrm{F}_{\mathrm{r}} / T \mathrm{P}_{\mathrm{f}}$

where TP is total protein concentration and $F_{r}$ is the fraction of alveolar radioactivity (obtained by $\gamma$-counting) in the lung at the end of the experiment.

KGF treatment. To maximize the rate of alveolar fluid clearance, some mice were treated with recombinant human KGF (Amgen Inc., Thousand Oaks, California, USA) by intratracheal administration 72 hours before clearance measurements, as done previously in rats (32). Maximum alveolar fluid clearance in mice was found at 72 hours (data not shown). A 24-gauge feeding tube was used to instill either KGF $(5 \mathrm{mg} / \mathrm{kg})$ in saline or saline alone $(2 \mathrm{~mL} / \mathrm{kg})$ into the trachea of methoxyflurane-anesthetized mice via the mouth. After the saline/KGF bolus, $0.3 \mathrm{~mL}$ of air was instilled into the trachea. After instillation, the mice were allowed to recover from anesthesia and were maintained in their cages for 72 hours before clearance measurements. KGF administration did not produce morbidity or mortality in any mouse.

Northern blot, immunoblot, and RT-PCR analysis. AQP5 transcript and protein expression in lung were evaluated by Northern blot using the AQP5 coding sequence as probe, and by immunoblot using specific polyclonal antibodies (21). Expression of transcripts encoding other lung aquaporins was evaluated semiquantitatively using RT-PCR. Peripheral lung cDNA was used as template for PCR amplification of the full-length coding sequences of the 4 mouse lung aquaporins, using primers flanking the coding sequences. Template concentration and the number of amplification cycles were adjusted so that the amount of amplified product was sensitive to transcript amount. Transcript amounts from different mice were normalized by amplification of a 500-bp fragment of $\beta$-actin.

\section{Results}

Figure 1a shows AQP5 transcript and protein expression in lungs of wild-type mice, AQP5 heterozygous mice, and AQP5 knockout mice. As expected, AQP5 transcript and protein were not detectable in the knockout mice. By quantitative immunoblot analysis, AQP5 protein expression was reduced by approximately $30 \%$ in AQP5 heterozygous mice compared with wild-type mice. The effect of AQP5 deletion on AQP1, AQP3, and AQP4 transcript expression was examined by semiquantitative RT-PCR (Figure 1b). AQP5 deletion did not affect transcript expression of the other lung aquaporins. Immunoperoxidase staining using specific antibodies showed no difference in the expression pattern of AQP1, AQP3, and AQP4 between wild-type and AQP5 knockout mice (not shown).

Osmotically driven water transport between the airspace and capillary compartments was measured by the established pleural surface fluorescence method using both increases ( 300 to $500 \mathrm{mOsm}$ ) and decreases (300 to $200 \mathrm{mOsm}$ ) in perfusate osmolality to drive water flow. Figure 2 shows original time-course data. In response to the addition of $200 \mathrm{mM}$ sucrose to the pulmonary artery perfusate, there was an increase in pleural surface fluorescence as the fluorescent dye in the airspaces was concentrated (left). Removal of the sucrose produced a reversible decrease in fluorescence with a similar time course. Fluorescence reversibly decreased and increased upon switching perfusate osmolalities

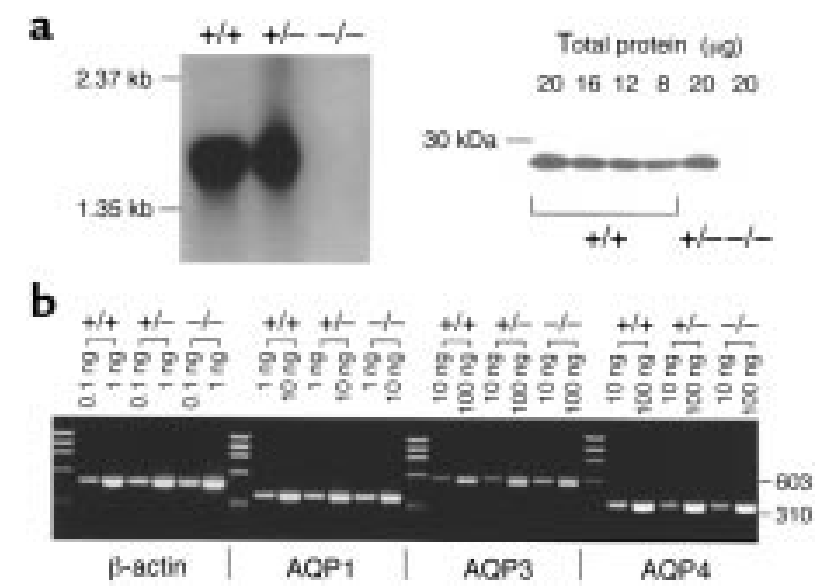

\section{Figure 1}

Aquaporin expression and localization in lungs of wild-type $(+/+)$ mice, AQP5 heterozygous (+/-) mice, and AQP5 knockout (-/-) mice. (a) Northern blot (left) and quantitative immunoblot analysis (right) of AQP5 expression in homogenates of whole lungs from mice of indicated genotypes. (b) RT-PCR analysis of aquaporin expression in mouse lungs. Fragments of $\beta$-actin, AQP1, AQP3, and AQP4 were amplified by PCR using specific primers and the indicated amounts of lung CDNA from mice with the indicated genotypes. 
between 300 and $200 \mathrm{mOsm}$ (right). The rate of fluorescence change provides a quantitative measure of $\mathrm{P}_{\mathrm{f}}$ of the airspace-capillary barrier. Changes in fluorescence were very rapid in wild-type mice as reported previously (4), with a $P_{\mathrm{f}}$ of $0.022 \mathrm{~cm} / \mathrm{s}$. Changes in fluorescence were slightly slowed in AQP5 heterozygous mice, but were remarkably slowed in AQP5-null mice. Averaged results are given in Figure 4.

Interestingly, the fluorescence curves for the AQP5null mice showed a biphasic behavior consisting of a small, prompt signal deflection (Figure 2, leftward arrows) followed by a slower change in the fluorescence making up the majority of the signal amplitude. As discussed previously (3), one source of a prompt signal deflection in this assay is a change in the light transmittance properties of the interstitium that occurs as a consequence of water movement between the capillary and interstitial compartments. Water permeability of the microvascular endothelial barrier in AQP5-null mice is high because AQP1 expression is unimpaired, so a change in perfusate osmolality should produce a prompt change in interstitial water content followed by a slower change in airspace fluid osmolality. This interpretation is supported by measurements of lungs from AQP1-null mice in which the small rapid signal deflection was never seen (Figure 2). Similarly, the rapid signal deflection was not observed in lungs from AQP1/AQP5 double-knockout mice, where water permeability was markedly reduced.

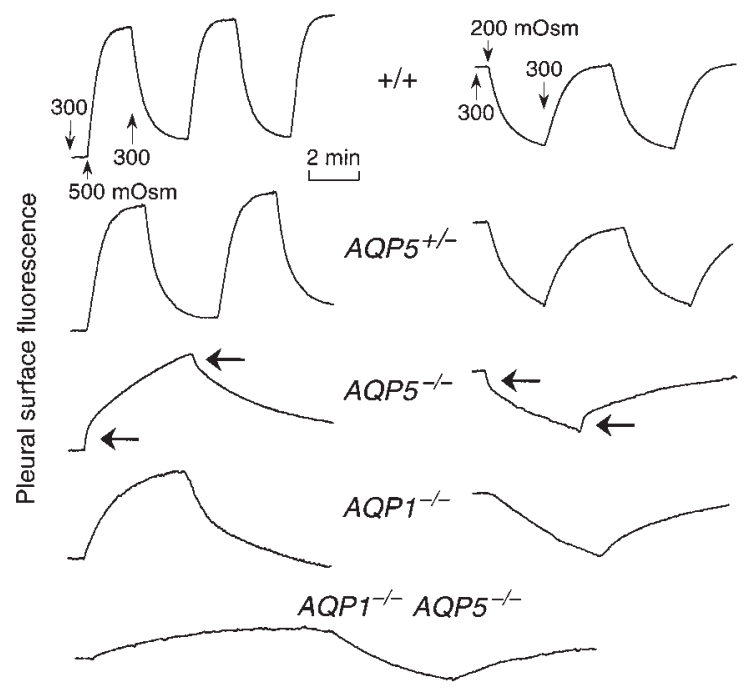

Figure 2

Airspace-capillary water permeability measured by a pleural surface fluorescence method. Isolated lungs were perfused with solutions of the indicated osmolalities, and the airspace was filled with an isosmolar solution containing FITC-dextran, as described in Methods. Representative time-course data shown for lungs of mice of indicated genotypes. Osmolalities were switched between $300 \mathrm{mOsm}$ and 500 mOsm (left), or $300 \mathrm{mOsm}$ and $200 \mathrm{mOsm}$ (right). Leftward arrows in data from AQP5 knockout mice point to an early phase of rapid signal change resulting from rapid transcapillary water transport.
Airspace-capillary $\mathrm{P}_{\mathrm{f}}$ was determined by an independent gravimetric method in which changes in lung weight were measured in response to changes in pulmonary artery perfusate osmolality. In contrast to the pleural surface fluorescence method, the gravimetric method measures water permeability in alveoli throughout the lung (not only at the pleural surface), and does not suffer from artifacts related to the optical properties of the interstitium. Figure 3 shows original lung weight curves after changes in perfusate osmolality. Rates of lung weight change related to osmotic water permeability were remarkably slowed by deletion of AQP5 and AQP1. The weight curves for lungs of AQP5-null mice sometimes showed a very small, prompt weight change (as discussed above), probably resulting from rapid changes in interstitial water content. Prompt weight changes were never seen in lungs from AQP1-null mice.

Figure 4 summarizes averaged $P_{f}$ values from a series of pleural surface fluorescence measurements (Figure 4a) and rates of lung weight change from a series of gravimetric measurements (Figure $4 \mathrm{~b}$ ). Both measurement methods show a remarkable 10 -fold decrease in airspace-capillary water permeability in AQP5 null mice, similar to that found in AQP1 null mice. Water permeability in AQP5 heterozygous mice was reduced by approximately $30 \%$ compared with wild-type mice. Water permeability was further reduced in AQP1/AQP5 double-knockout mice.

Active alveolar fluid absorption was measured in an in situ lung model from the increased concentration of an impermeant volume indicator ( ${ }^{125} \mathrm{I}$-albumin) instilled into the airspace at zero time. A 15-minute endpoint was used because active alveolar fluid clearance was found to be linear over this time. Figure 5a shows rapid alveolar fluid clearance (12\% in 15 minutes) in control wild-type mice that was increased to $18 \%$ in 15 minutes when $\beta$-agonist (isoproterenol or terbutaline) was present in the instillate. Alveolar fluid clearance was blocked by the transport inhibitors amiloride and ouabain, indicating an active transport process involving $\mathrm{Na}^{+}$channels and $\mathrm{Na}^{+} / \mathrm{K}^{+}$pumps. The large percentage of inhibition caused by amiloride in mouse lung has been noted previously (8), and is probably related to the dominance of the epithelial $\mathrm{Na}^{+}$ channel $(\mathrm{ENaC})$ as the principal pathway for apical membrane $\mathrm{Na}^{+}$transport. Similar measurements done in mice treated for 72 hours with KGF, an agent causing type II cell proliferation $(20,33)$, showed an alveolar clearance rate of $28 \%$ in 15 minutes with isoproterenol stimulation.

Alveolar fluid clearance measurements were made in wild-type mice and AQP5 knockout mice in the presence of isoproterenol to maximally stimulate fluid absorption and to eliminate uncertainties about endogenous catechol concentration. Figure $5 \mathrm{~b}$ shows that alveolar fluid absorption did not differ between litter-matched wild-type mice and AQP5null mice. There was little variability in clearance rate from mouse to mouse. An additional set of compar- 


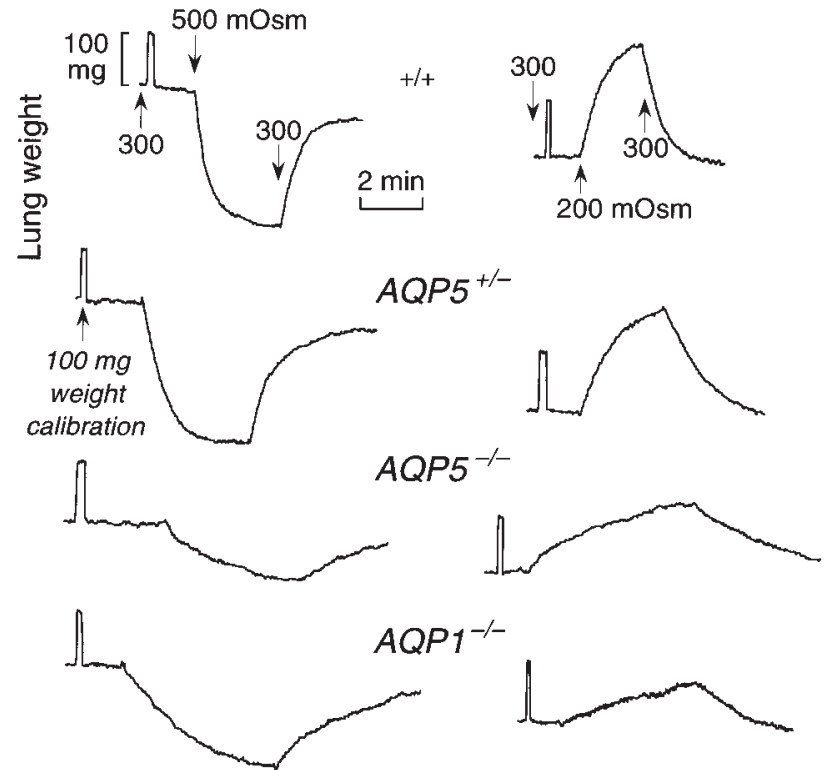

Figure 3

Airspace-capillary water permeability measured by a gravimetric method. Isolated lungs were perfused, as in Figure 2, and lung weight was measured continuously by a gravimetric transducer, as described in Methods. Representative time-course data shown are for lungs of mice of indicated genotypes. Pulmonary artery perfusate solutions were switched as indicated. A 100-mg weight calibration was done at the beginning of each experiment.

ative measurements was done in mice treated for 72 hours with KGF to increase alveolar fluid clearance even more. Although there was more variability in alveolar clearance rates in the isoproterenol-stimulated lungs from KGF-treated mice, there was again no difference in average fluid clearance between wildtype and AQP5 knockout mice (Figure 5b, right). Extravascular lung water content was measured at the end of each experiment by determination of lung wet-to-dry weight ratios in which intravascular lung water was corrected by hemoglobin assay of lung homogenates and peripheral blood. Expressed as corrected wet-to-dry weight ratios, AQP5 deletion did not affect extravascular lung water content. Mean \pm SEM (in milligrams) for the mice represented in Figure $5 \mathrm{~b}$ were $9.0 \pm 0.3$ (wild-type), $9.2 \pm 0.4$ (AQP5 knockout), $8.6 \pm 0.6$ (wild-type, $+\mathrm{KGF}$ ), and $8.8 \pm 0.3$ (AQP5 knockout, $+\mathrm{KGF}$ ). These results indicate that active alveolar fluid absorption was not affected despite a 10-fold decrease in airspace-capillary $\mathrm{P}_{\mathrm{f}}$ in AQP5-deficient mice.

Finally, increasing lung edema in response to a hydrostatic driving force was measured gravimetrically. Lungs were gravity perfused with an isosmolar saline solution at a constant left atrial outflow pressure of $5 \mathrm{~cm} \mathrm{H}_{2} \mathrm{O}$ and at different pulmonary artery perfusate pressures. Our previous measurements were done at an outflow pressure of 0 (transected, noncannulated left atrium), which produced relatively small and saturable changes in lung weight
(24). Maintenance of left atrial pressure at $5 \mathrm{~cm} \mathrm{H}_{2} \mathrm{O}$ produced a greater increase in lung weight in response to increases in inflow pressure. Figure 6a shows representative weight curves in which inflow pressure was increased from $8 \mathrm{~cm} \mathrm{H}_{2} \mathrm{O}$ to $18 \mathrm{~cm} \mathrm{H}_{2} \mathrm{O}$, and then to $38 \mathrm{~cm} \mathrm{H}_{2} \mathrm{O}$. In response to an increase in pressure to $38 \mathrm{~cm} \mathrm{H}_{2} \mathrm{O}$, there was an approximately 5 -fold increase in filtration initially (note different ordinate scale), followed by more rapid lung water accumulation accompanied by overt alveolar flooding with fluid overflow from the trachea. Qualitatively similar rates of lung water accumulation were found in wild-type and AQP5-null mice. The quantitative data summary in Figure $6 \mathrm{~b}$ indicates that AQP5 deletion does not affect hydrostatically driven lung water accumulation (filtration).

\section{Discussion}

The 10-fold decrease in the $\mathrm{P}_{\mathrm{f}}$ of the airspace-capillary barrier in AQP5-deficient mice indicates that AQP5 provides the principal pathway for water movement across the apical surface of the alveolar epithelium. Because AQP5 deletion should affect transcellular but not paracellular water permeability, the reduced water permeability in AQP5-null mice indicates that the primary route for water movement across the alveolar epithelium is transcellular and through type I cells. The minimal residual water permeability across the alveolar apical surface after AQP5 deletion may involve type
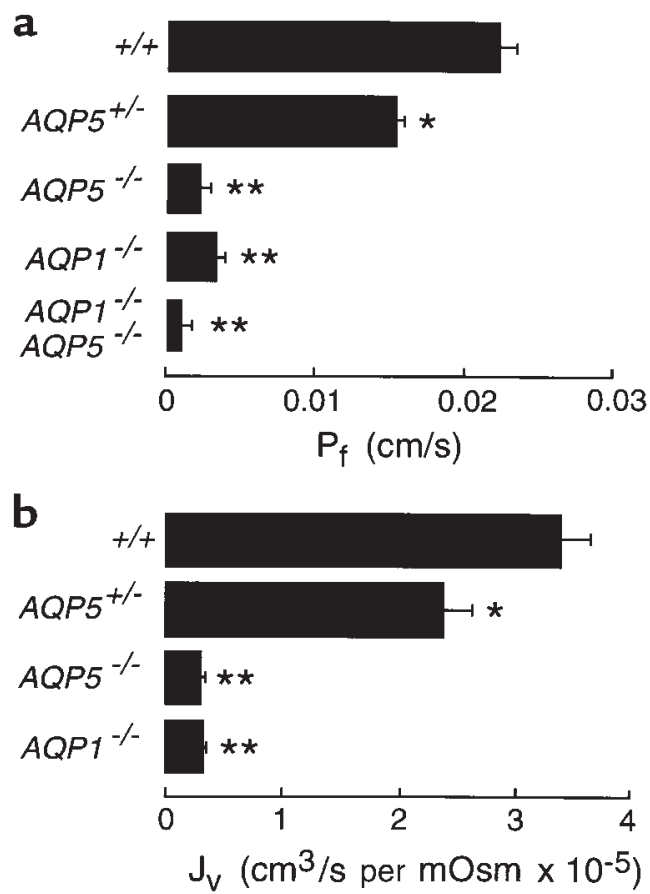

Figure 4

Averaged airspace-capillary osmotic water permeability (mean \pm $\mathrm{SEM}, n=7-10$ ) determined from surface fluorescence measurements (a) and rates of lung weight $\left(J_{v}\right)$ change in $\mathrm{cm}^{3} / \mathrm{s}$ per $\mathrm{mOsm}$, del 1 determined from gravimetric measurements $(\mathbf{b}) .{ }^{*} P<0.01 \mathrm{vs}$. wild type $(+/+){ }^{* *} P<0.0001$ vs. wild type. 
a

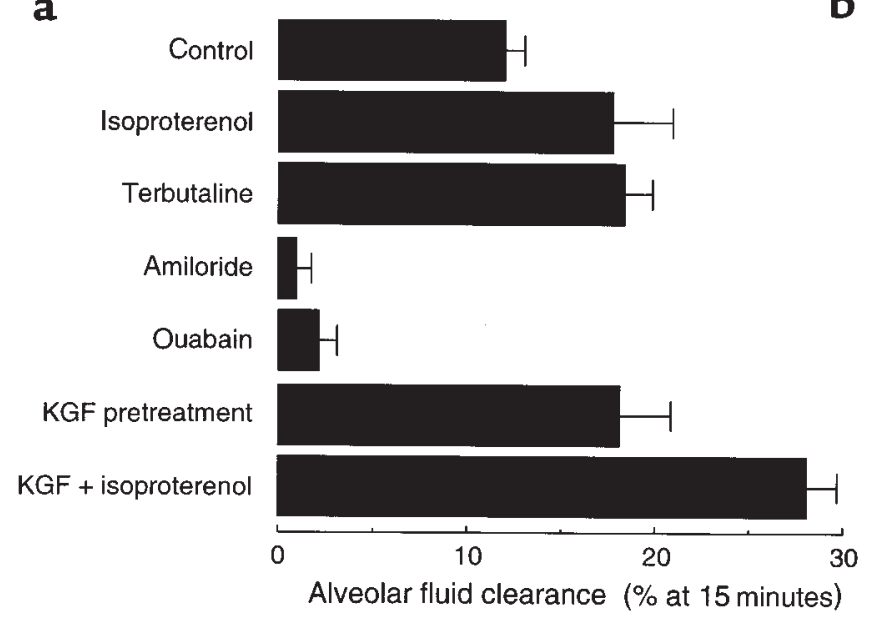

b

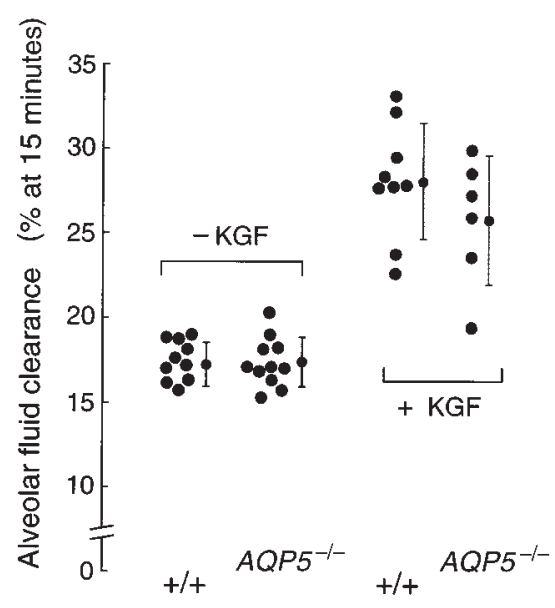

Figure 5

Effect of AQP5 deletion on active alveolar fluid clearance. Measurements were done at $37^{\circ} \mathrm{C}$ using an in situ perfused lung preparation in which the airspace was instilled with an isosmolar solution containing ${ }^{131} \mathrm{l}$-albumin as a volume marker. Alveolar fluid clearance is expressed as percentage fluid absorption (mean $\pm \mathrm{SD}, n=6-10$ mice) at 15 minutes. (a) Measurements of lungs from wild-type mice. Where indicated, the instillate contained isoproterenol $(0.1 \mathrm{mM})$, terbutaline $(10 \mathrm{mM})$, amiloride $(1 \mathrm{mM})$, or ouabain $(0.1 \mathrm{mM})$, or measurements were performed 72 hours after treatment of mice with KGF (see Methods). (b) Measurements on lungs from litter-matched wild-type mice and AQP5 knockout mice. Data from individual mice (filled circles) and averaged (mean \pm SD) values are shown. The instillate contained $1 \mathrm{mM}$ isoproterenol in all experiments. Where indicated, mice were pretreated with KGF.

II alveolar epithelial cells, the apical membrane lipid of type I cells, and possibly as-yet unidentified water transporters. Because the alveolar apical surface becomes the rate-limiting barrier for airspace-capillary water transport in AQP5 knockout mice, the $\mathrm{P}_{\mathrm{f}}$ of 0.002 $\mathrm{cm} / \mathrm{s}$ in AQP5 knockout mice is approximately equal to the $\mathrm{P}_{\mathrm{f}}$ of the (hypothetically smooth) apical plasma membrane of AQP5-deficient type I cells. Because the type I cell apical membrane is highly invaginated (5, 34), the intrinsic apical membrane $\mathrm{P}_{\mathrm{f}}$ of AQP5-deficient cells is less than $0.001 \mathrm{~cm} / \mathrm{s}$, which is consistent with the water permeability of lipid membranes not containing water channels. The $\mathrm{P}_{\mathrm{f}}$ of isolated rat type I cells in suspension, where water moves across both apical and basolateral plasma membranes, was $0.07 \mathrm{~cm} / \mathrm{s}(5)$. Assuming a similar $\mathrm{P}_{\mathrm{f}}$ in type I cells from mice, these results suggest that the basolateral membrane has greater water permeability than does the apical membrane. No aquaporins have yet been located in the basolateral membrane of type I cells, raising the possibility of as-yet unidentified aquaporin or nonaquaporin water-transporting proteins in lung. Homology cloning may provide a useful strategy to identify new aquaporin-type water channels using lung cDNA from triple-knockout mice lacking AQP1, AQP4, and AQP5, or quadruple-knockout mice using recently created AQP3-null mice (35). We also note that transcripts encoding the recently identified water channels AQP9 (36) and AQP9L (37) are expressed at low levels in lung, and thus represent candidates for the alveolar basolateral membrane water pathway.

An important function of the alveolar epithelium is the active clearance of fluid from the airspaces. Alveolar fluid absorption contributes to the resolution of pul- monary edema in many clinical settings. Fluid absorption across the alveolar epithelium involves active salt transport, which is energized by basolateral membrane $\mathrm{Na}, \mathrm{K}-\mathrm{ATP}$ ase and requires apical membrane $\mathrm{Na}^{+}$channels ( $\mathrm{ENaC}$ and non-ENaC proteins) and possibly $\mathrm{Cl}^{-}$ transporters. Recent data indicates that adenovirusmediated delivery of the $\mathrm{Na}$,K-ATPase $\beta 1$ subunit gene can upregulate alveolar fluid clearance in rats (38). Water transport is driven by osmotic gradients created by salt transport by a presumed near-isosmolar transport mechanism in which small osmotic gradients drive water movement across the highly water-permeable alveolar epithelium. We reported that AQP1 deletion in kidney proximal tubule inhibits active fluid absorption from the tubule lumen (22), and that AQP5 deletion in salivary gland inhibits active fluid secretion into the lumen of serous acini (29). The saliva produced by AQP5 knockout mice was hypertonic because of salt secretion into the acinar lumen without adequate amounts of water. From these mechanistic considerations, it has been postulated that high alveolar epithelial water permeability facilitates active airspace fluid absorption.

Active airspace fluid absorption was compared in wild-type and AQP5 knockout mice using an in situ mouse model (39). The rate of $\beta$-agonist-stimulated alveolar fluid absorption in this model (more than 1\% per minute) is higher than maximal rates of lung fluid absorption in rat and larger animal models (8). Fluid absorption rates of nearly $2 \%$ per minute were attained in KGF-pretreated, $\beta$-agonist-stimulated mice. Our mouse model with very rapid active alveolar fluid absorption thus provides a sensitive test of whether high alveolar epithelial water permeability is required for active fluid absorption. It was found that AQP5 dele- 
tion, which decreased alveolar epithelial water permeability 10-fold, had no significant effect on alveolar fluid clearance, even under maximal KGF induction and $\beta$-agonist stimulation (32). There are several explanations for this finding. First, the rate of airspace fluid absorption is substantially less than the rate of active fluid absorption (per unit surface area) in proximal tubule and the rate of active fluid secretion in the pilocarpine-stimulated salivary gland acinus. In the latter two systems, active fluid transport exceeds $0.5 \mu \mathrm{L} / \mathrm{min}$ per square micrometer of epithelial surface area $(22,29)$. Using a mouse lung epithelial surface area of $600 \mathrm{~cm}^{2}$ (40), an active fluid absorption rate of $10 \mu \mathrm{L} / \mathrm{min}$ (equivalent to $30 \%$ absorption in 15 minutes) gives a rate of $0.016 \mu \mathrm{L} / \mathrm{min}$ per square micrometer, substantially lower than that in kidney proximal tubule and salivary gland. Slower rates of active fluid transport probably do not require such high cell membrane water permeability (30). Another consideration is that in the alveolus, unlike the kidney proximal tubule and salivary gland, salt and water move primarily through different cells - type II and type I cells, respectively. Therefore, 3compartment models using solute-solvent coupling to accomplish isosmolar fluid transport probably do not apply to the alveolar epithelium. Another difference between active fluid transport in alveolus vs. kidney proximal tubule and salivary gland is that fluid is rapidly cleared on both sides of the epithelium in kidney (by lumen and capillary flow) and salivary gland (by capillary flow and saliva expulsion from the acinus), whereas fluid moves relatively slowly in the alveolar airspaces.

Our gravimetric measurements indicated that AQP5 deletion did not impair the accumulation of lung edema driven by increased hydrostatic pressure. The experimental conditions were selected so as to produce alveolar edema because AQP5 is located on the alveolar epithelium and thus would not contribute to interstitial edema involving fluid movement from the capillary to interstitial compartments across the microvascular endothelium. Mechanistically, it is understandable why AQP5 should not facilitate hydrostatically driven fluid movement across the relatively tight alveolar epithelial barrier. AQP5 appears to be a water-selective transporter with unity reflection coefficients for solutes $(41,42)$. Therefore, solute-free water driven into the airspace compartment by hydrostatic forces, unless accompanied by solute entry, would be opposed by strong osmotic driving forces. It is believed that the accumulation of alveolar edema involves transient breakdown of the integrity of the alveolar barrier, permitting the entry of solutes and protein. Therefore, bulk movement of fluid through rifts in the alveolar barrier would not be expected to involve AQP5 as found here.

Our results thus raise questions about the role of AQP5 in lung physiology. Although AQP5 provides a quantitatively important route for osmotically driven water movement into and out of the airspaces, it does not appear to facilitate hydrostatically driven lung edema or active alveolar fluid absorption. Furthermore, the minimal expression of AQP5 in lung at the time of birth, and the delayed appearance of AQP5 after birth suggests that AQP5 is not involved in perinatal alveolar fluid clearance (16-18). The tissue-specific expression of a specific aquaporin by no means proves its physiological significance, as we have found for AQP4 expression in gastric parietal cells and skeletal muscle plasmalemma. Although AQP5 is required for salivary gland fluid secretion, the expression of AQP5 in alveolar type I cells and the very high alveolar epithelial water permeability may represent an evolutionary remnant with little physiological importance. Our data do not rule out the possibility that AQP5 is involved in other lung functions such as gas exchange, maintenance of the alveolar fluid subphase, and alveolar epithelial cell volume regulation; however, it is not clear how an apical membrane water-transporting protein might be involved in these processes. Last, it is possible that AQP5 might function in concert with other water or ion transporters. Demonstration of an overt phenotype in transgenic mice might require generation of double- or multiple-knockout mice lacking AQP5 as well as other proteins.
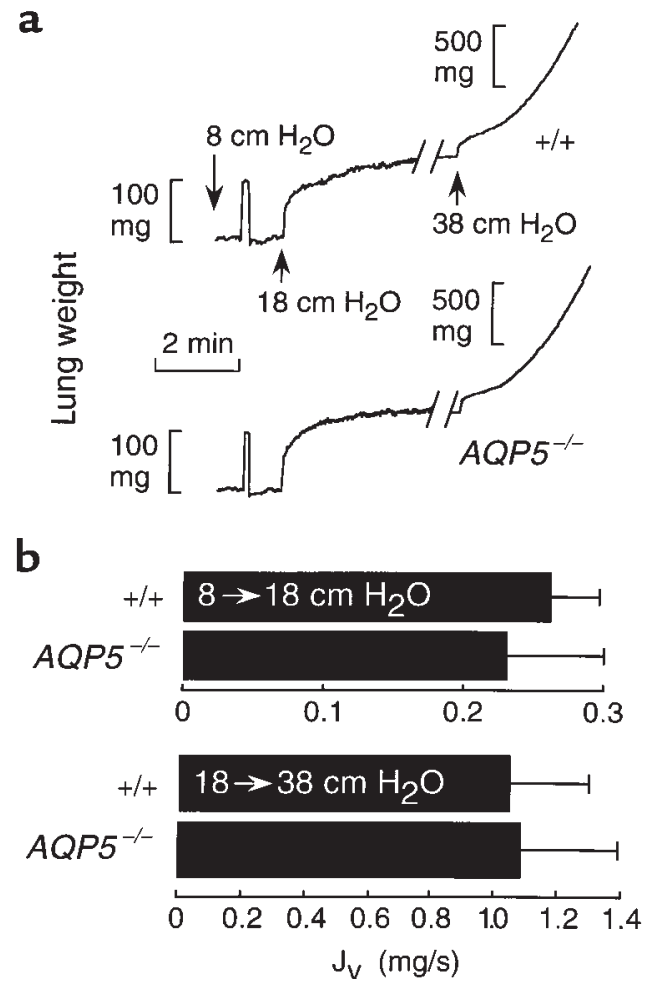

\section{Figure 6}

Gravimetric measurement of hydrostatically driven lung edema (filtration). Weight was monitored continuously in isolated lungs perfused with an isosmolar solution at indicated hydrostatic pressures set by adjusting reservoir height. The left atrium was cannulated, and outflow pressure was fixed at $5 \mathrm{~cm} \mathrm{H}_{2} \mathrm{O}$. (a) Representative weight curves for mice of indicated genotypes. (b) Averaged filtration rates (mean \pm SEM, $n=5-7$ mice in each group) at 1-3 minutes after increasing pulmonary artery pressure from $8 \mathrm{~cm} \mathrm{H}_{2} \mathrm{O}$ to $18 \mathrm{~cm} \mathrm{H}_{2} \mathrm{O}$ and just after increasing pressure from $18 \mathrm{~cm} \mathrm{H}_{2} \mathrm{O}$ to $38 \mathrm{~cm} \mathrm{H}_{2} \mathrm{O}$. No significant difference was detected between wild-type and $\mathrm{AQP5} 5^{-/-}$mice. 


\section{Acknowledgments}

We thank Liman Qian for transgenic mouse breeding and genotype analysis. This study was supported by National Institutes of Health grants HL-59198, HL51854, DK-35124, HL-60288, and DK-43840, and grant R613 from the National Cystic Fibrosis Foundation.

1. Verkman, A.S. 1998. Water transporting mechanisms in airways and lung. In Pulmonary edema. M.A. Matthay and D. Ingbar, editors. Marcel Dekker Inc. New York, NY. 525-547.

2. Folkesson, H.G., Matthay, M.A., Hasegawa, H., Kheradmand, F., and Verkman, A.S. 1994. Transcellular water transport in lung alveolar epithelium through mercury-sensitive water channels. Proc. Natl. Acad. Sci. USA. 91:4970-4974.

3. Carter, E.P., Matthay, M.A., Farinas, J., and Verkman, A.S. 1996 Transalveolar osmotic and diffusional water permeability in intact mouse lung measured by a novel surface fluorescence method. J. Gen Physiol. 108:133-142.

4. Carter, E.P., Umenishi, F., Matthay, M.A., and Verkman, A.S. 1997. Developmental changes in alveolar water permeability in perinatal rabbit lung. J. Clin. Invest. 100:1071-1078.

5. Dobbs, L., et al. 1998. Highly water-permeable type I alveolar epithelial cells confer high water permeability between the airspace and vasculature in rat lung. Proc. Natl. Acad. Sci. USA. 95:2991-2996.

6. Carter, E.P., Ölveczky, B.P., Matthay, M.A., and Verkman, A.S. 1998. High microvascular endothelial water permeability in mouse lung measured by a pleural surface fluorescence method. Biophys. J. 74:2121-2128.

7. Folkesson, H., Matthay, M., Frigeri, A., and Verkman, A.S. 1996. High transepithelial water permeability in microperfused distal airways: evidence for channel-mediated water transport. J. Clin. Invest. 97:664-671.

8. Matthay, M.A., Folkesson, H., and Verkman, A.S. 1996. Salt and water transport across alveolar and distal airway epithelia in the adult lung. Am. J. Physiol. 270:L487-L503.

9. Olver, R.E. 1994. Fluid secretion and absorption in the fetus. In Fluid and solute transport in the airspaces of the lung. R.M. Effros and H.K. Chang, editors. Marcel Dekker Inc. New York, NY. 281-302.

10. Nielsen, S., King, L.S., Christensen, B.M., and Agre, P. 1997. Aquaporins in complex tissues. II. Subcellular distribution in respiratory and glandular tissues of rat. Am. J. Physiol. 273:C1549-C1561.

11. Hasegawa, H., Zhang, R., Dohrman, A., and Verkman, A.S. 1993. Tissuespecific expression of mRNA encoding the rat kidney water channel CHIP28k by in situ hybridization. Am. J. Physiol. 264:C237-C245.

12. Effros, R.M., et al. 1997. Water transport and distribution of aquaporin1 in the pulmonary airspaces. J. Appl. Physiol. 83:1002-1016.

13. Frigeri, A., Gropper, M., Turck, C.W., and Verkman, A.S. 1995. Immunolocalization of the mercurial-insensitive water channel and glycerol intrinsic protein in epithelial cell plasma membranes. Proc. Natl. Acad. Sci. USA. 92:4328-4331.

14. King, L.S., Nielsen, S., and Agre, P. 1997. Aquaporins in complex tissues. I. Developmental patterns in respiratory and glandular tissues of rat. Am. J. Physiol. 273:C1541-C1548.

15. Funaki, H., et al. 1998. Localization and expression of AQP5 in cornea, serous salivary glands, and pulmonary epithelial cells. Am. J. Physiol. 275:C1151-C1157.

16. Umenishi, F., et al. 1996. Sharp increase in rat lung water channel expression in the perinatal period. Am. J. Respir. Cell Mol. Biol. 15:673-679.

17. Yasui, M., et al. 1997. Perinatal changes in expression of aquaporin-4 and other water and ion transporters in rat lung. J. Physiol. 505:3-11.

18. Ruddy, M.K., Drazen, J.M., Pitkanan, O.M., Rafii, B., and Harris, H.W. 1998. Modulation of aquaporin-4 and the amiloride-inhibitable sodium channel in perinatal rat lung epithelial cells. Am. J. Physiol. 274:L1066-L1072.

19. King, L.S., Nielsen, S., and Agre, P. 1996. Aquaporin-1 water channel protein in lung. Ontogeny, steroid-induced expression, and distribution in rat. J. Clin. Invest. 97:2183-2191.

20. Borok, Z., et al. 1998. Keratinocyte growth factor modulates alveolar epithelial cell phenotype in vitro: expression of aquaporin 5. Am. J. Respir Cell Mol. Biol. 18:554-561.

21. Ma, T., et al. 1998. Severely impaired urinary concentrating ability in transgenic mice lacking aquaporin-1 water channels. J. Biol. Chem. 273:4296-4299.

22. Schnermann, J., Chou, J., Ma, T., Knepper, M.A., and Verkman, A.S. 1998 Defective proximal tubule reabsorption in transgenic aquaporin-1 null mice. Proc. Natl. Acad. Sci. USA. 95:9660-9664.

23. Chou, C.L., et al. 1999. Reduced water permeability and altered ultrastructure in thin descending limb of Henle in aquaporin-1 null mice. J. Clin. Invest. 103:491-496.

24. Bai, C., et al. 1999. Lung fluid transport in aquaporin-1 and aquaporin 4 knockout mice. J. Clin. Invest. 103:555-561.

25. Ma, T., et al. 1997. Generation and phenotype of a transgenic knockout mouse lacking the mercurial-insensitive water channel aquaporin-4. J. Clin. Invest. 100:957-962.

26. Chou, C.L., Ma, T., Yang, B., Knepper, M.A., and Verkman, A.S. 1998. Four-fold reduction in water permeability in inner medullary collecting duct of aquaporin-4 knockout mice. Am. J. Physiol. 274:C549-C554.

27. Manley, G.T., et al. 2000. Aquaporin-4 deletion in mice reduces brain edema following acute water intoxication and ischemic stroke. Nat. Medicine. In press.

28. Matsuzaki, T., Suzuki, T., Koyama, H., Tanaka, S., and Takata, K. 1999 Aquaporin-5 (AQP5), a water channel protein, in the rat salivary and acrimal glands: immunolocalization and effect of secretory stimulation. Cell Tissue Res. 295:513-521.

29. Ma, T., et al. 1999. Defective secretion of saliva in transgenic mice lacking aquaporin-5 water channels. J. Biol. Chem. 274:20071-20074.

30. Spring, K.R. 1998. Routes and mechanism of fluid transport by epithelia. Annu. Rev. Physiol. 60:105-119.

31. Rezaiguia, S., et al. 1997. Acute bacterial pneumonia in rats increases alveolar epithelial fluid clearance by tumor necrosis factor-alphadependent mechanism. J. Clin. Invest. 99:325-335.

32. Wang, Y., Folkesson, H.G., Jayr, C., Ware, L.B., and Matthay, M.A. 1999. Alveolar epithelial fluid transport can be simultaneously upregulated by both KGF and beta-agonist therapy. J. Appl. Physiol. 87:1852-1860.

33. Ulich, T.R., et al. 1994. Keratinocyte growth factor is a growth factor for type II pneumocytes in vivo. J. Clin. Invest. 93:1298-1306.

34. Stone, K.C., Mercer, R.R., Gehr, P., Stockstill, B., and Crapo, J.D. 1992 Distribution of cell numbers and volumes between alveolar and non alveolar tissue. Am. J. Respir. Cell Mol. Biol. 6:235-243.

35. Ma, T., et al. 1999. Targeted gene deletion of aquaporin-3 in mice. J. Am. Soc. Nephrol. 10:19A

36. Ishibashi, K., et al. 1998. Cloning and functional expression of a new aquaporin (AQP9) abundantly expressed in the peripheral leukocytes permeable to water and urea, but not glycerol. Biochem. Biophys. Res. Comm. 244:268-274.

37. Ko, S.B., et al. 1999. Cloning and functional expression of rAQP9L, a new member of aquaporin family from rat liver. Biochem. Mol. Biol. Int. 47:309-318.

38. Factor, P., et al. 1998. Augmentation of lung liquid clearance via adenovirus-mediated transfer of $\mathrm{Na}, \mathrm{K}-\mathrm{ATP} a s e$ subunit gene. J. Clin. Invest. 102:1421-1430.

39. Garat, C., Carter, E., and Matthay, M.A. 1998. New in situ mouse model to quantify alveolar epithelial fluid clearance. J. Appl. Physiol. 84:1763-1767.

40. Wangensteen, O.D. 1994. Nonselective solute transport across the pulmonary epithelium. In Fluid and solute transport in the airspaces of the lung. Volume 70. R.M. Effros and H.K. Chang., editors. 374-397.

41. Yang, B., and Verkman, A.S. 1997. Water and glycerol permeability of aquaporins 1-5 and MIP determined quantitatively by expression of epitopetagged constructs in Xenopus oocytes. J. Biol. Chem. 272:16140-16146.

42. Meinild, A.K., Klaerke, D.A., and Zeuthen, T. 1998. Bidirectional water fluxes and specificity for small hydrophilic molecules in aquaporins 0 5. J. Biol. Chem. 273:32446-32451. 